\title{
Relación entre la percepción de validez de una rúbrica, el rendimiento académico y la autorregulación de estudiantes de ciencias del deporte.
}

Irene Jimenez-Perez ${ }^{\text {a,b }}$, Lara Requena-Bueno ${ }^{a}$, Marina Gil-Calvo ${ }^{a}$, Pedro Pérez-Soriano ${ }^{\mathbf{a}}$ y Jose Ignacio Priego-Quesada ${ }^{\mathrm{a}, \mathrm{b}}$

${ }^{a}$ Grupo de Investigación en Biomecánica aplicada al Deporte (GIBD), Departamento de Educación Física y Deportiva, Facultad de Ciencias de la Actividad Física y el Deporte, Universitat de València, ${ }^{\text {b}}$ Grupo de Investigación en Física Médica (GIFIME), Departamento de Fisiología, Facultad de Medicina y Odontología, Universitat de València. Emails: i.jimenez.gibd@gmail.com; 1.requena.gibd@gmail.com; m.gil.gibd@gmail.com; pedro.perez-soriano@uv.es; j.ignacio.priego@uv.es

\section{\$ EWWDFW}

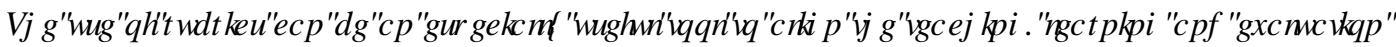

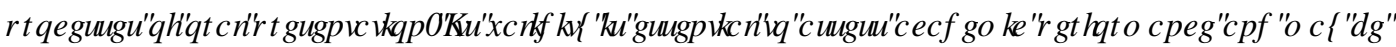

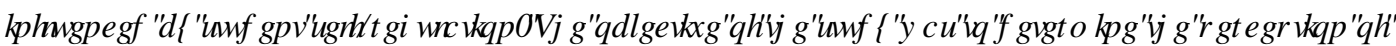

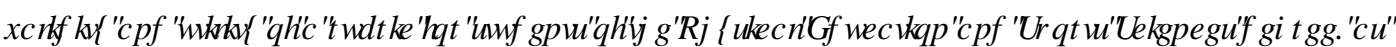

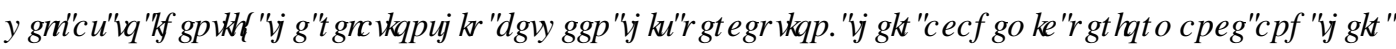

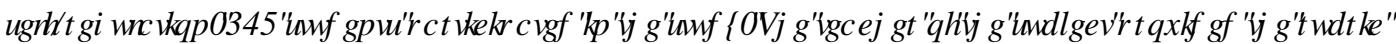

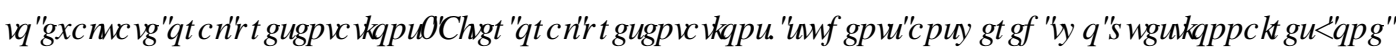

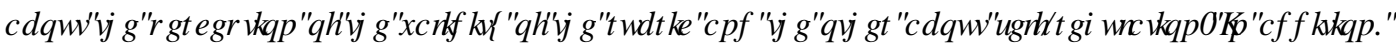

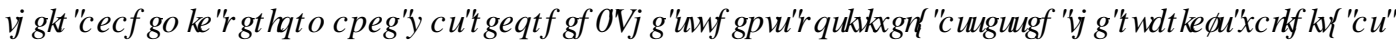

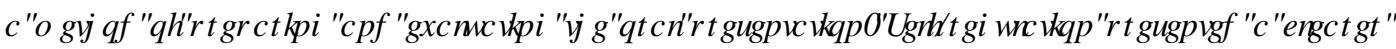

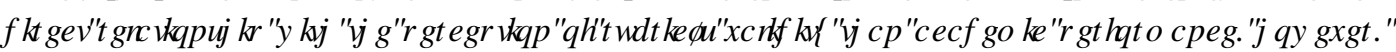

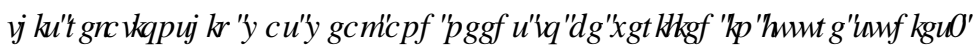
]

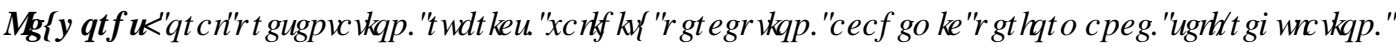
KJJKHUHCXFDURQIODQQDUTHDODDRQW

(

प

\section{HXP HQ]}

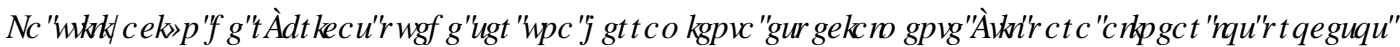

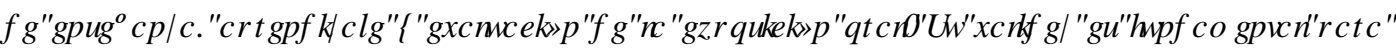

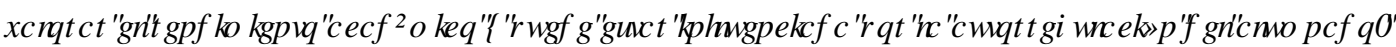
( ORENAMWR GHDHMGIR] KD FRQMUGG

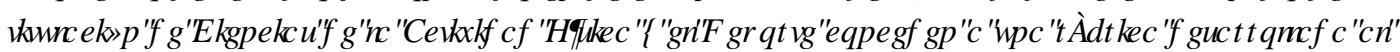

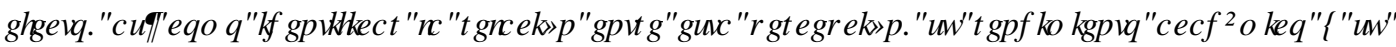

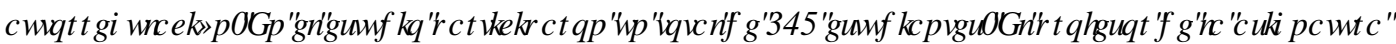

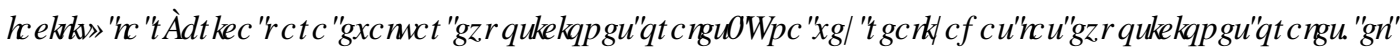

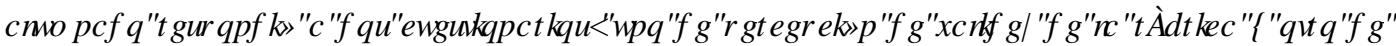

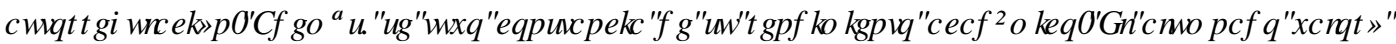




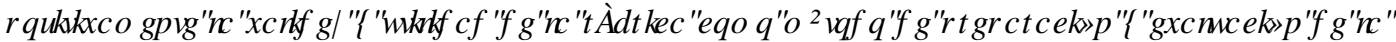
HSRUFIY QRLDOV DDXURUH XФFIYQSUHHQWXQDUHDFIYQGUFWDP

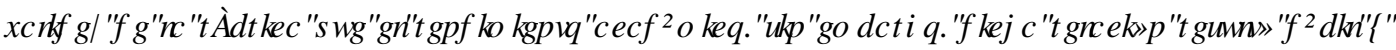
QHFMWDUHIFRQWDWDODQIXWLRVHMXGRW

(

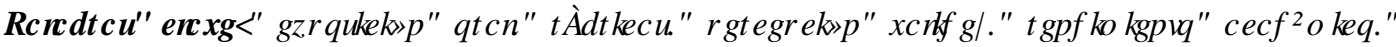

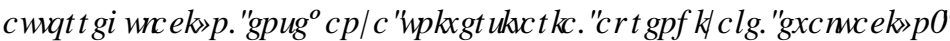




\section{Introducción}

La potenciación de competencias comunicativas es uno de los objetivos del nuevo modelo formativo de enseñanza universitaria para preparar a los estudiantes para sus roles futuros en su lugar de trabajo (Živković 2014, Verano-Tacoronte $H W D O$ 2016). La exposición oral es el medio más utilizado para desarrollar estas competencias comunicativas, sin embargo, su enseñanza y aprendizaje es una tarea compleja por los múltiples aspectos a tratar (de contenido, formales, de tiempo, etc.) y por la variedad de criterios de evaluación aplicables (Dasí e Iborra 2007; Verano-Tacoronte HMDO2016). En este contexto, cabe la necesidad de unificar criterios y apreciaciones, y para ello, la elaboración y utilización de rúbricas resulta fundamental como herramienta de evaluación de estas competencias, pues permite alinear los procesos de enseñanza, aprendizaje y evaluación de las mismas (Dunbar HWDO2006).

Las rúbricas se definen como guías que articulan los criterios usados para evaluar la calidad de las elaboraciones y el nivel de ejecución alcanzado por los estudiantes, especificando con antelación los factores y variables que se van a analizar y los niveles de cumplimiento en cada uno de ellos (Jonsson and Svingby 2007, Reddy and Andrade 2010). Así, las rúbricas proporcionan ciertos beneficios: a) promover un aprendizaje de mayor calidad, al centrar su atención en los criterios positivos; b) mejorar la autoevaluación de los estudiantes; c) facilitar a los docentes la emisión de juicios más válidos; d) proporcionar mejor retroalimentación al alumnado, y e) mejorar la consistencia entre las valoraciones de los evaluadores (Jonsson and Svingby 2007, García-Ros 2011).

Aunque las rúbricas son un método cada vez más extendido en la enseñanza obligatoria, todavía es escasa su consideración en el contexto universitario (García-Ros 2011). El principal motivo parece ser la reticencia sobre su fiabilidad y validez para valorar el rendimiento académico del alumnado (Reddy and Andrade 2010). En este sentido, en diferentes estudios se ha visto que la utilización de rúbricas ha sido bien valorada por el alumnado (Andrade and Du 2005, Barney HMDO 2011, Eshun and Osei-Poku 2013), sin embargo, también se ha encontrado que gran parte de ellos no observó una mejora de su rendimiento, sino que consiguió realizar la tarea según la demanda del profesor (Andrade and Du 2005). Por ello, ante la aplicación de una rúbrica, se hace necesario conocer la percepción del alumnado sobre la validez y utilidad de la misma. Además, también parece imprescindible analizar si la aplicación de dicha rúbrica proporciona una mejora directa del rendimiento académico. En este sentido, en el curso pasado se observó cómo entre dos grupos de la misma asignatura, el grupo que obtuvo una mayor calificación final en la asignatura fue el que también tuvo una percepción de la rúbrica más positiva (Priego Quesada HWDO2019).

Por último, otro beneficio a destacar de la utilización de rúbricas es que facilitan la autorregulación del aprendizaje de los estudiantes (Nicol and Macfarlane-Dick 2006), sin embargo, su efectividad parece depender del diseño de la misma (Panadero and Romero 2014). Por lo tanto, es posible que la percepción de la validez de la rúbrica por parte del alumnado presente una estrecha relación con su autorregulación, lo cual ha sido poco estudiado hasta el momento. 


\section{Objetivos}

El estudio pretendió determinar la percepción de validez que conceden los estudiantes universitarios de Ciencias de la Actividad Física y del Deporte a una rúbrica dirigida a evaluar la calidad de sus exposiciones orales, identificando adicionalmente la relación entre la percepción de validez, el rendimiento académico y su autorregulación.

\section{Desarrollo de la innovación}

\subsection{Alumnado sobre el que se ha desarrollado la intervención}

La intervención se llevó a cabo en el curso 2019-2020, en la asignatura "Metodología de la Enseñanza de la Actividad Física y el Deporte" del Grado de Ciencias de la Actividad Física y el Deporte, de la Universitat de València. Dicha asignatura se imparte en el primer cuatrimestre del tercer curso del grado. El alumnado se divide en dos grupos según el idioma de impartición de la materia, castellano y valenciano, teniendo cada uno de éstos 50 y 73 estudiantes matriculados, respectivamente, en el presente curso analizado.

\subsection{Diseño de la intervención}

La intervención se puso en práctica en el tema de la asignatura donde se desarrollan los diferentes estilos de enseñanza aplicables a las ciencias de la actividad física y el deporte. En este tema, el profesor imparte tres clases y deja el resto de sesiones para que los alumnos presenten los diferentes estilos de enseñanza mediante exposiciones orales. En la primera clase, el profesor realizó una introducción del tema. En la segunda entrenó al alumnado en el uso de la rúbrica y le involucró en su proceso de modificación. Y en la tercera proporcionó conclusiones para cerrar el tema. Entre la segunda y tercera clase, se llevaron a cabo las exposiciones orales por parte del alumnado. Los grupos de castellano y valenciano realizaron un total de 8 y 10 exposiciones orales, respectivamente. La normativa de las exposiciones orales consistió en los siguientes puntos:

- La composición de los equipos la realizó el profesor siguiendo un criterio de orden alfabético en la lista.

- Los equipos tenían un número de componentes de entre 5 y 7.

- La duración de la exposición debía ser entre 25 y 35 minutos.

- El mismo día, justo antes de la exposición, el equipo debía dar un folio al profesor con la división de la exposición según el número de componentes, y el profesor decidir qué miembro debía exponer cada una de las partes. El objetivo era que todos los componentes tuviesen que conocer la presentación al completo.

- En cada presentación oral, tanto el profesor como el alumnado que no realizaba la exposición, debían evaluar al equipo mediante la utilización de una rúbrica (sección 3.3). La nota final de la exposición oral fue del $60 \%$ por parte del profesor, del $30 \%$ por parte del alumnado, y un $10 \%$ en forma de autoevaluación.

- Antes de que el alumnado conociese su calificación obtenida, se les pidió que cumplimentaran un cuestionario anónimo sobre la percepción de validez de la rúbrica (sección 3.4) y sobre su autorregulación (sección 3.6). 
En la sesión en la que se realizó el entrenamiento sobre el uso de la rúbrica, el procedimiento seguido fue el siguiente: en primer lugar, se proporcionó la rúbrica al alumnado y visualizaron un vídeo de una exposición oral que tuvieron que valorar mediante el empleo de la rúbrica. Tras esa primera valoración, pudieron preguntar las dudas que tuviesen sobre los criterios de evaluación considerados en la rúbrica, así como sobre los niveles de ejecución considerados en los mismos y la estrategia de calificación utilizada. A continuación, se les dio adicionalmente la opción de modificar los distintos componentes destacados (criterios, niveles de ejecución y estrategia de calificación). Así, en cada ítem se fue debatiendo si modificar o no alguno de sus aspectos, aunque ambos grupos decidieron finalmente no realizar ninguna modificación. Tras este proceso, se visualizó un segundo vídeo y lo volvieron a evaluar utilizando la rúbrica.

La distribución temporal de la intervención se muestra en la Tabla 1.

Tabla 1. Distribución temporal de la intervención.

\begin{tabular}{cc}
\hline Momento & Fecha \\
\hline Entrenamiento rúbrica & $10 / 10 / 2019$ \\
$\begin{array}{c}\text { Realización exposiciones orales } \\
\text { Realización cuestionario percepción validez } \\
\text { rúbrica y autorregulación }\end{array}$ & Del 22/10/2019 al 07/11/2019 \\
Publicación notas exposición oral & $07 / 11 / 2019$ \\
\hline
\end{tabular}

\subsection{Rúbrica}

Se utilizó como rúbrica para valorar la exposición oral, una versión modificada de la desarrollada y evaluada por García-Ros (2011) (Tabla 2). 
Tabla 2. Rúbrica utilizada para las exposiciones orales.

\begin{tabular}{|c|c|c|c|c|}
\hline \multicolumn{5}{|c|}{ 1. Principios básicos $(30 \%)$} \\
\hline & Inadecuado 0 & A mejorar 1 & Adecuado 2 & Muy adecuado 3 \\
\hline $\begin{array}{c}\text { Dominio y } \\
\text { comprensión del } \\
\text { tema }\end{array}$ & $\begin{array}{c}\text { No domina ni comprende el } \\
\text { tema }\end{array}$ & $\begin{array}{c}\text { Muestra una buena comprensión } \\
\text { de partes del tema, pero no de } \\
\text { alguna de ellas }\end{array}$ & $\begin{array}{c}\text { Muestra una buena comprensión } \\
\text { del tema }\end{array}$ & $\begin{array}{l}\text { Manifiesta una comprensión } \\
\text { completa y profunda del tema }\end{array}$ \\
\hline $\begin{array}{l}\text { Planificación y } \\
\text { organización del } \\
\text { contenido }\end{array}$ & No ha planificado & $\begin{array}{c}\text { Discurso difícil de seguir y } \\
\text { conexiones incoherentes, lo que } \\
\text { indica planificación pobre y que } \\
\text { ha dedicado poco esfuerzo }\end{array}$ & $\begin{array}{c}\text { Sigue un esquema general } \\
\text { adecuado, aunque alguna vez se } \\
\text { pierde o necesita prestar más } \\
\text { atención a detalles }\end{array}$ & $\begin{array}{l}\text { Discurso bien planificado, } \\
\text { enlaza los tópicos de manera } \\
\text { lógica y coherente }\end{array}$ \\
\hline \multicolumn{5}{|c|}{ 2. Aplicación y ejemplificación $(30 \%)$} \\
\hline & Inadecuado 0 & A mejorar 1 & Adecuado 2 & Muy adecuado 3 \\
\hline $\begin{array}{l}\text { Coherencia con el } \\
\text { modelo teórico }\end{array}$ & $\begin{array}{l}\text { El ejemplo desarrollado no es } \\
\text { coherente con el modelo }\end{array}$ & $\begin{array}{l}\text { El ejemplo desarrollado es } \\
\text { coherente con el modelo, } \\
\text { aunque diversos aspectos no } \\
\text { quedan recogidos } \\
\text { adecuadamente }\end{array}$ & $\begin{array}{c}\text { El ejemplo desarrollado es } \\
\text { coherente con el modelo teórico, } \\
\text { aunque necesitaría afinar algún } \\
\text { aspecto concreto }\end{array}$ & $\begin{array}{c}\text { El ejemplo desarrollado es } \\
\text { coherente con el modelo teórico }\end{array}$ \\
\hline $\begin{array}{l}\text { Clarificación } \\
\text { modelo y } \\
\text { motivación } \\
\text { audiencia }\end{array}$ & $\begin{array}{c}\text { El ejemplo seleccionado es } \\
\text { confuso y no se ajusta al } \\
\text { modelo }\end{array}$ & $\begin{array}{l}\text { El ejemplo no clarifica } \\
\text { adecuadamente y no resulta } \\
\text { motivante para la audiencia }\end{array}$ & $\begin{array}{c}\text { El ejemplo no clarifica } \\
\text { adecuadamente o resulta } \\
\text { motivante para la audiencia }\end{array}$ & $\begin{array}{c}\text { El ejemplo resulta clarificador y } \\
\text { motivante para la audiencia }\end{array}$ \\
\hline \multicolumn{5}{|c|}{ 3. Apoyo visual $(20 \%)$} \\
\hline & Inadecuado 0 & A mejorar 1 & Adecuado 2 & Muy adecuado 3 \\
\hline $\begin{array}{l}\text { Número y } \\
\text { explicación de las } \\
\text { diapositivas }\end{array}$ & $\begin{array}{l}\text { No cumple requisitos de número } \\
\text { y adecuación en su explicación. }\end{array}$ & $\begin{array}{l}\text { Utiliza un número inadecuado de } \\
\text { diapositivas, aunque intenta } \\
\text { explicarlas }\end{array}$ & $\begin{array}{c}\text { Cantidad de diapositivas } \\
\text { adecuada, aunque imprecisa } \\
\text { explicación de alguna de ellas }\end{array}$ & $\begin{array}{l}\text { Cantidad de diapositivas } \\
\text { adecuada y explicación } \\
\text { pertinente de las mismas }\end{array}$ \\
\hline $\begin{array}{l}\text { Adecuación de } \\
\text { las diapositivas }\end{array}$ & $\begin{array}{l}\text { Incorpora diapositivas no } \\
\text { pertinentes con la temática, no } \\
\text { facilitan la comprensión ni la } \\
\text { motivación de la audiencia. }\end{array}$ & $\begin{array}{l}\text { Aunque la mayoría de ellas } \\
\text { están relacionadas con el tema, } \\
\text { no favorecen su comprensión ni } \\
\text { la motivación de la audiencia }\end{array}$ & $\begin{array}{l}\text { Todas tiene relación con el tema y } \\
\text { facilitan que la comprensión } \\
\text { material, aunque algunas no están } \\
\text { conectadas con el discurso o } \\
\text { adolecen de amenidad }\end{array}$ & $\begin{array}{l}\text { Todas tiene relación con el } \\
\text { tema y conexión con el } \\
\text { discurso, facilitando la } \\
\text { comprensión del material y } \\
\text { resultan amenas/captan la } \\
\text { atención }\end{array}$ \\
\hline Legibilidad & $\begin{array}{c}\text { Ilegible, uso de colores } \\
\text { abrumador, abusa de texto y de } \\
\text { párrafos }\end{array}$ & $\begin{array}{c}\text { Letra legible por el } 85 \% \\
\text { audiencia aproximad., extensión } \\
\text { excesiva en gran parte } \\
\text { diapositivas. Inadecuado } \\
\text { contraste de colores }\end{array}$ & $\begin{array}{c}\text { Letra legible para toda la } \\
\text { audiencia, más de } 5 \text { líneas por } \\
\text { diapositiva. Contraste adecuado de } \\
\text { colores }\end{array}$ & $\begin{array}{l}\text { No más de } 5 \text { líneas por } \\
\text { diapositiva, letra legible para } \\
\text { toda la audiencia, contraste } \\
\text { adecuado de colores }\end{array}$ \\
\hline $\begin{array}{c}\text { Relevancia y } \\
\text { adecuación } \\
\text { imágenes/esque } \\
\text { mas }\end{array}$ & No utiliza o distraen & $\begin{array}{l}\text { Acorde con contenido, aunque } \\
\text { no relevantes y de poca } \\
\text { resolución }\end{array}$ & $\begin{array}{c}\text { Relevantes, acordes con } \\
\text { contenidos en la } \\
\text { mayoria de ocasiones, pero poca } \\
\text { resolución }\end{array}$ & $\begin{array}{c}\text { Relevantes, acordes con } \\
\text { contenidos y con adecuada } \\
\text { resolución }\end{array}$ \\
\hline
\end{tabular}

(c)) EY-NC-ND 2021, Universitat Politècnica de València

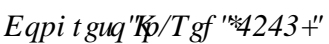




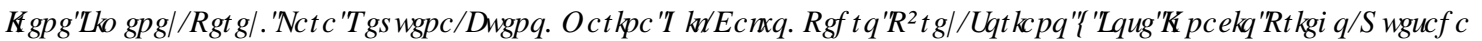

\begin{tabular}{|c|c|c|c|c|}
\hline $\begin{array}{l}\text { Secuenciación, } \\
\text { animaciones, } \\
\text { transiciones } \\
\text { diapositivas }\end{array}$ & $\begin{array}{l}\text { Ningún orden, excesivo o } \\
\text { ningún uso } \\
\text { animación y transición que } \\
\text { facilita distracción }\end{array}$ & $\begin{array}{c}\text { Orden lógico, aunque falta } \\
\text { animación y sólo aplica } \\
\text { transiciones en alguna } \\
\text { diapositiva, o efectúa ambas de } \\
\text { forma inadecuada }\end{array}$ & $\begin{array}{l}\text { Orden lógico, animación de puntos } \\
\text { no importantes o transiciones que } \\
\text { dificultan atención }\end{array}$ & $\begin{array}{l}\text { Animación puntos importantes, } \\
\text { ayuda a centrar la atención en } \\
\text { lo importante y evita distraerse }\end{array}$ \\
\hline \multicolumn{5}{|c|}{ 4. Habilidades de comunicación $(20 \%)$} \\
\hline & Inadecuado 0 & A mejorar 1 & Adecuado 2 & Muy adecuado 3 \\
\hline $\begin{array}{c}\text { Volumen y tono } \\
\text { de voz }\end{array}$ & $\begin{array}{l}\text { Demasiado bajo para ser } \\
\text { escuchado por todos y } \\
\text { excesivamente monótono }\end{array}$ & $\begin{array}{l}\text { Suficientemente alto para ser } \\
\text { escuchado alrededor del } 70 \% \\
\text { del tiempo por todos y/o tono } \\
\text { inadecuado (monótono, } \\
\text { aburrido) }\end{array}$ & $\begin{array}{l}\text { Suficientemente alto para ser } \\
\text { escuchado por todos y tono de voz } \\
\text { adecuado alrededor del } 90 \%\end{array}$ & $\begin{array}{l}\text { Suficientemente alto y con tono } \\
\text { de voz adecuado para ser } \\
\text { escuchado por toda la audiencia }\end{array}$ \\
\hline $\begin{array}{l}\text { Claridad en el } \\
\text { habla }\end{array}$ & $\begin{array}{l}\text { Poca claridad. A menudo } \\
\text { masculla y pronuncia mal } \\
\text { palabras }\end{array}$ & $\begin{array}{c}\text { Habla claro } 85-90 \% \text { del tiempo y } \\
\text { pronuncia mal numerosas } \\
\text { palabras }\end{array}$ & $\begin{array}{c}\text { Habla claro el } 90-95 \% \text { del tiempo, } \\
\text { aunque se equivoca al pronunciar } \\
\text { algunas palabras }\end{array}$ & $\begin{array}{l}\text { Habla claro la práctica totalidad } \\
\text { del tiempo. }\end{array}$ \\
\hline $\begin{array}{l}\text { Pausas y uso de } \\
\text { coletillas }\end{array}$ & $\begin{array}{l}\text { No usa pausas de manera } \\
\text { intencionada Abuso de } \\
\text { coletillas. Gran cantidad de } \\
\text { frases incompletas }\end{array}$ & $\begin{array}{l}\text { Número de pausas inadecuado - } \\
\text { por exceso o defecto-, introduce } \\
\text { algunas en momentos } \\
\text { inesperados o utiliza } \\
\text { frecuentemente coletillas (um..., } \\
\text { ehhh..., bien...etc). Utiliza } \\
\text { muchas frases incompletas }\end{array}$ & $\begin{array}{c}\text { Usa las pausas correctamente, } \\
\text { aunque introduce algunas de forma } \\
\text { inesperada/ inadecuada, utiliza en } \\
\text { diversas ocasiones coletillas } \\
\text { (um..., ehhh..., bien...etc). Utiliza } \\
\text { frases incompletas en más del } 5 \% \\
\text { ocasiones }\end{array}$ & $\begin{array}{c}\text { Usa las pausas correctamente y } \\
\text { al final de las oraciones. Utiliza } \\
\text { frases completas en más del } \\
95 \% \text { ocasiones }\end{array}$ \\
\hline $\begin{array}{c}\text { Postura y } \\
\text { contacto visual }\end{array}$ & $\begin{array}{l}\text { No mantiene contacto visual y la } \\
\text { postura corporal es inadecuada }\end{array}$ & $\begin{array}{l}\text { A veces mantiene postura } \\
\text { erguida y mantiene contacto } \\
\text { visual con la audiencia, pero } \\
\text { menos del } 90 \% \text { del tiempo }\end{array}$ & $\begin{array}{c}\text { Postura erguida y establece } \\
\text { contacto visual con todos, aunque } \\
\text { se observan algunos indicadores } \\
\text { de tensión/desinterés }\end{array}$ & $\begin{array}{c}\text { Espalda recta, postura relajada } \\
\text { y confiada, establece contacto } \\
\text { visual con todos }\end{array}$ \\
\hline \multicolumn{5}{|c|}{ 5. Innovación y creatividad $(+0,5)$} \\
\hline
\end{tabular}

(c) BY-NC-ND 2021, Universitat Politècnica de València

\&RQJ UHR Q Q5 HGपिए०० 


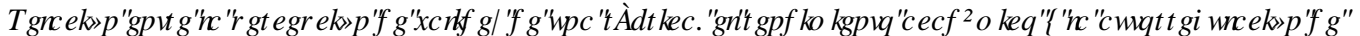

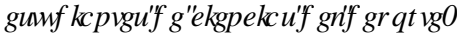

\subsection{Cuestionario de percepción de validez de la rúbrica}

Se desarrolló un cuestionario dirigido a evaluar la percepción de los estudiantes sobre la validez y utilidad de la rúbrica. Este cuestionario estuvo compuesto por 9 ítems (ver ítems en Tabla 4), utilizando una escala de respuesta tipo Likert de 5 niveles, desde "nada de acuerdo" hasta "muy de acuerdo". Además, se consideró la puntuación total de percepción de validez como la suma de las puntuaciones de todos los ítems. El cuestionario fue realizado por un total de 85 participantes, de forma anónima. El cuestionario fue validado previamente (Priego Quesada HMDO2019).

\subsection{Valoración del rendimiento académico}

Se utilizaron las calificaciones obtenidas por los distintos grupos de estudiantes en las exposiciones orales, tanto en los cinco subapartados de la rúbrica (principios básicos, aplicación y ejemplificación, apoyo visual, habilidades de comunicación, e innovación y creatividad), como en la valoración final, así como la calificación final de la asignatura. Dichas calificaciones se utilizaron como valores de control y discusión de ambos grupos.

\subsection{Cuestionario de autorregulación}

Se empleó el cuestionario "Emotion and Motivation Self-Regulation Questionnaire" (EMSR-Q) de AlonsoTapia $H W D O(2014)$ para analizar la autorregulación del alumnado. Este cuestionario incluye 20 ítems que se responden en una escala tipo Likert de 5 niveles, desde "nunca" hasta "siempre". Los ítems se agrupan en dos escalas: autorregulación del aprendizaje (con 8 ítems) y autorregulación del rendimiento/evitación (con 12 ítems) (Tabla 3). La primera escala incluye acciones que están orientadas a objetivos de aprendizaje, por lo que cuanto mayor sea el valor en esta escala, más positivo será el efecto de la autorregulación en el aprendizaje. La segunda escala incluye acciones que muestran falta de autorregulación u orientación al rendimiento, por lo que cuanto mayor sea el valor en esta escala, más negativas serán las acciones para el rendimiento del aprendizaje.

Tabla 3. Ítems del cuestionario de autorregulación (EMSR_Q).

\begin{tabular}{|l|l|}
\hline \multicolumn{2}{|c|}{ Autorregulación del aprendizaje $(\alpha=0,90)$} \\
\hline 1 & ¡Esto va bien! ... Parece que lo entiendo. \\
\hline 2 & Cálmate ... .No te apures, no pares” ... Lo conseguirás. \\
\hline 3 & Bueno ... Parece que cada vez lo hago mejor ... estoy progresando... \\
\hline 4 & ¡Qué interesante! Parece que lo entiendo. \\
\hline 5 & ¡Es difícil, pero qué interesante! ... Tengo que entender cómo hacerlo. \\
\hline 6 & Esto no está bien ... voy a verificarlo paso a paso. \\
\hline 7 & Que complicado ... Bueno, continuaré ... es importante aprender a resolverlo. \\
\hline 8 & ¡Aquí estaba el error! ¡Excelente! La próxima vez sé cómo hacerlo. \\
\hline \multicolumn{2}{|c|}{ Autorregulación del rendimiento/evitación $(\alpha=0,88)$} \\
\hline 9 & No vale la pena mi tiempo ... Intentaré terminarlo lo antes posible. \\
\hline 10 & ¡Esta tarea es una completa pérdida de tiempo! \\
\hline 11 & ¡Qué instrucciones tan largas! Lo único que hacen es confundirme. \\
\hline 12 & ¡Qué tarea tan aburrida! A ver si termino y me voy. \\
\hline 13 & Estoy muerto de cansancio ... Bueno, tengo que seguir para aprobar. \\
\hline 14 & Debo continuar ... si no lo hago, fracasaré. \\
\hline
\end{tabular}




\begin{tabular}{|l|l|}
\hline 15 & ¡Qué desastre! Bueno ... tengo que continuar ... si no, no aprobaré el examen. \\
\hline 16 & ¡Qué tarea tan agotadora! ... Pero tengo que aprobar ... tengo que continuar. \\
\hline 17 & ¡Qué tarea estresante! Lo estoy haciendo muy mal ... ¡Es muy difícil! \\
\hline 18 & Esto es muy difícil ... no voy a poder hacerlo bien. \\
\hline 19 & No estoy hecho para hacer esto. Si pudiera, lo abandonaría. \\
\hline 20 & Me estoy poniendo nervioso ... no puedo hacerlo. \\
\hline
\end{tabular}

\subsection{Análisis estadístico}

Para el análisis estadístico se utilizó el programa SPSS (SPSS Statistics v.26, IBM, Nueva York, EEUU). Debido a que durante el curso 2018/2019 se utilizó la rúbrica original diseñada por García-Ros (2011) y para para el curso de la presente intervención (2019/2020) se incluyeron algunas modificaciones, se han comparado los valores de percepción de la validez de la rúbrica entre ambos cursos en cada uno de los ítems mediante pruebas T-Student para muestras independientes. En dichas comparaciones se utilizó p <0,05 como límite de significación estadística. Con el objetivo de analizar las relaciones entre la percepción de validez, el rendimiento académico y la autorregulación, se utilizó el análisis de correlaciones bivariadas mediante la $r$ de Pearson.

\section{Resultados}

\subsection{Percepción de la validez y utilidad de la rúbrica}

Debido a que durante el curso 2018/2019 se utilizó la rúbrica original diseñada por García-Ros (2011) y para el curso de la presente intervención (2019/2020) se incluyeron algunas modificaciones, se han comparado los valores de percepción de la validez de la rúbrica entre ambos cursos (Tabla 4). Antes que nada, se puede observar como durante ambos cursos la rúbrica fue valorada muy positivamente obteniendo en la mayoría de los casos valores promedio superiores al 4 (correspondiente a las valoraciones entre "De acuerdo" y "Muy de acuerdo").

Se observan ligeras diferencias entre la valoración de la rúbrica original y la modificada en este curso. Sin embargo, al haberse evaluado ambas rúbricas por alumnado diferente, se considera que los presentes datos están objetivando que no hay mejora ni prejuicio notables. Las diferencias observadas podrían constatarse realizando un estudio en el que el mismo grupo utilizase ambas rúbricas y las valorase. 
Tabla 4. Media y desviación estándar (DE) de la valoración de la percepción de validez de la rúbrica por parte

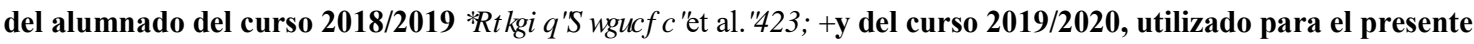
estudio.

\begin{tabular}{lccc}
\hline \multicolumn{1}{c}{ Media (DE) sobre un máximo de 5 puntos } & & $\begin{array}{c}\text { Dif entre ambos } \\
\text { cursos }\end{array}$ \\
\hline \multicolumn{1}{c}{ Ítem } & $\mathbf{2 0 1 8 / 2 0 1 9}$ & $\mathbf{2 0 1 9 / 2 0 2 0}$ & $\mathbf{p}$ \\
\hline $\begin{array}{l}\text { 1.Me ha parecido muy apropiada para valorar las } \\
\text { exposiciones. }\end{array}$ & $4,2(0,5)$ & $4,3(0,6)$ & 0,35 \\
\hline $\begin{array}{l}\text { 2.Me ha parecido muy fácil de utilizar. } \\
\text { 3.El número de ítems era adecuado. }\end{array}$ & $4,4(0,7)$ & $4,5(0,7)$ & 0,41 \\
\hline $\begin{array}{l}\text { 4.Era fácil en cada ítem saber la valoración de cada } \\
\text { grupo. }\end{array}$ & $3,0(0,9)$ & $4,1(0,9)$ & 0,63 \\
\hline $\begin{array}{l}\text { 5.Teniendo la rúbrica es más fácil preparar una } \\
\text { exposición para conseguir buena nota. }\end{array}$ & $4,6(0,6)$ & $4,0(0,7)$ & $<\mathbf{0 . 0 0 1}$ \\
\hline $\begin{array}{l}\text { 6.Con la rúbrica se puede ser más justo en la } \\
\text { evaluación. }\end{array}$ & $4,2(0,7)$ & $4,3(0,8)$ & 0,61 \\
\hline $\begin{array}{l}\text { 7.La rúbrica nos ha ayudado a preparar la } \\
\text { exposición. }\end{array}$ & $4,3(0,9)$ & $\mathbf{0 1}(0,7)$ & $\mathbf{0 , 0 2}$ \\
\hline $\begin{array}{l}\text { 8.La rúbrica recoge los aspectos más importantes } \\
\text { a evaluar de la exposición. }\end{array}$ & $4,2(0,7)$ & $3,9(1,0)$ & 0,30 \\
\hline $\begin{array}{l}\text { 9.Recomiendo que se siga utilizando la rúbrica en } \\
\text { futuros cursos. }\end{array}$ & $4,4(0,7)$ & $4,1(0,7)$ & 0,68 \\
\hline $\begin{array}{l}\text { 10.Valoración total (suma de todos los ítems; } \\
\text { valoración máxima= 45). }\end{array}$ & $37,9(3,7)$ & $4,4(0,6)$ & 0,82 \\
\hline
\end{tabular}

\subsection{Relación entre la valoración de la rúbrica, el rendimiento académico y la autorregulación}

Se han observado diferentes resultados entre la percepción de la rúbrica y las siguientes variables analizadas (Tabla 5):

- Rendimiento académico: No se observó una relación clara entre la percepción de validez de la rúbrica y el rendimiento académico. En primer lugar, ninguna variable de percepción de validez se relacionó con la nota final de la asignatura. El alumnado que obtuvo una mayor nota en la exposición oral sí que valoró más positivamente el uso de la rúbrica para conseguir una mejor nota. Sin embargo, el alumnado con mejor expediente académico valoró más negativamente el uso de la rúbrica como método para valorar las exposiciones, así como su facilidad de uso. En cualquier caso, todas las relaciones observadas fueron débiles ( $\mathrm{r}=0.2 \mathrm{o}-0.3)$, y además no fueron consistentes ni repetidas en múltiples ítems, por lo que puede que estos resultados se deban al azar.

- Autorregulación del aprendizaje: Se observó una relación directa entre la autorregulación del aprendizaje y la percepción de validez de la rúbrica en 4 ítems, relacionados con: el número de ítems, la obtención de una nota más justa, su recomendación para futuros cursos, y la suma total de todos los ítems. Dichas relaciones también fueron débiles al igual que con el rendimiento académico $(\mathrm{r}=0,3)$. Sin embargo, el hecho de que en este caso se repitiesen en tres ítems y también en la suma total de los ítems proporciona una mayor consistencia al resultado. Por lo tanto, al igual que en estudios previos (Nicol and Macfarlane-Dick 2006), la autorregulación del aprendizaje sí parece tener una relación directa con la percepción de validez de la rúbrica.

- Autorregulación del rendimiento/evitación: No se observó ninguna relación entre la percepción de validez de la rúbrica y la autorregulación del rendimiento. Este resultado fue positivo ya que 
puede estar indicando que la rúbrica es de fácil uso, y no es peor o mejor valorada por el alumnado que tiene una peor o mejor autorregulación del rendimiento.

Tabla 5. Correlación de Pearson entre la valoración de la rúbrica, el rendimiento académico y la autorregulación.

\begin{tabular}{|c|c|c|c|c|c|}
\hline \multirow[b]{2}{*}{ Ítem } & \multicolumn{5}{|c|}{$R$ de Pearson ( $p$ valor) } \\
\hline & $\begin{array}{l}\text { Nota } \\
\text { asignatura }\end{array}$ & $\begin{array}{l}\text { Nota } \\
\text { exp. oral }\end{array}$ & $\begin{array}{c}\text { Nota } \\
\text { expediente }\end{array}$ & $\begin{array}{l}\text { Autorr. } \\
\text { aprendizaje }\end{array}$ & $\begin{array}{l}\text { Autorr. } \\
\text { rendimiento }\end{array}$ \\
\hline $\begin{array}{l}\text { 1.Me ha parecido muy apropiada para } \\
\text { valorar las exposiciones. }\end{array}$ &,$- 15(, 16)$ &, $01(, 94)$ &,$- 28(, 01)$ &, $08(, 45)$ & ,01 $(, 90)$ \\
\hline 2.Me ha parecido muy fácil de utilizar. &,$- 15(, 16)$ &,$- 04(, 74)$ &,$- 24(, 03)$ &, $08(, 45)$ &,$- 06(, 59)$ \\
\hline 3.El número de ítems era adecuado. &,$- 05(, 64)$ &, $01(, 96)$ &,$- 19(, 08)$ & ,22(,04) &,$- 04(, 74)$ \\
\hline $\begin{array}{l}\text { 4.Era fácil en cada ítem saber la } \\
\text { valoración de cada grupo. }\end{array}$ &, $04(, 73)$ &, $01(, 97)$ &,$- 12(, 29)$ &, $10(, 34)$ &,$- 04(, 72)$ \\
\hline $\begin{array}{l}\text { 5.Teniendo la rúbrica es más fácil } \\
\text { preparar una exposición para conseguir } \\
\text { buena nota. }\end{array}$ &, $04(, 69)$ &, $22(, 04)$ &,$- 10(, 39)$ &, $19(, 08)$ &,$- 01(, 93)$ \\
\hline $\begin{array}{l}\text { 6.Con la rúbrica se puede ser más justo en } \\
\text { la evaluación. }\end{array}$ &, $01(, 92)$ &, $04(, 71)$ &,$- 13(, 23)$ & ,24 (,03) &,$- 03(, 79)$ \\
\hline $\begin{array}{l}\text { 7.La rúbrica nos ha ayudado a preparar la } \\
\text { exposición. }\end{array}$ &, $06(, 56)$ &, $13(, 25)$ &, $06(, 60)$ &, $13(, 23)$ &,$- 07(, 52)$ \\
\hline $\begin{array}{l}\text { 8.La rúbrica recoge los aspectos más } \\
\text { importantes a evaluar de la exposición. }\end{array}$ &,$- 18(, 11)$ &,$- 15(, 16)$ &,$- 02(, 86)$ & ,20 (,07) &,$- 12(, 26)$ \\
\hline $\begin{array}{l}\text { 9. Recomiendo que se siga utilizando la } \\
\text { rúbrica en futuros cursos. }\end{array}$ &,$- 14(, 20)$ &,$- 14(, 21)$ &,$- 16(, 16)$ &, $34(, 002)$ &,$- 09(, 41)$ \\
\hline $\begin{array}{l}\text { 10.Valoración total (suma de todos los } \\
\text { ítems; valoración máxima }=45 \text { ). }\end{array}$ &,$- 08(, 47)$ &, $03(, 79)$ &,$- 20(, 07)$ & ,29 (,008) &,$- 08(, 45)$ \\
\hline
\end{tabular}

\section{Conclusiones}

La rúbrica es una herramienta que es valorada positivamente por el alumnado como método de preparación y evaluación de la exposición oral. La autorregulación del aprendizaje tuvo una relación directa más clara con la percepción de validez de la rúbrica que el rendimiento académico. En cualquier caso, dicha relación fue débil, y futuros estudios deberían analizar mediante un cuestionario mucho más amplio, cuáles son los principales factores que afectan la percepción de validez de la rúbrica del alumnado.

\section{Referencias}

ALONSO-TAPIA, J., CALDERÓN, E.P., AND RUIZ, M.A.D., (2014). "Development and Validity of the Emotion and Motivation Self-Regulation Questionnaire (EMSR-Q)" en The Spanish Journal of Psychology, 17.

ANDRADE, H. y DU, Y., (2005). "Student Perspectives on Rubric-Referenced Assessment" en Practical Assessment, Research, and Evaluation, vol.10, issue 3. 


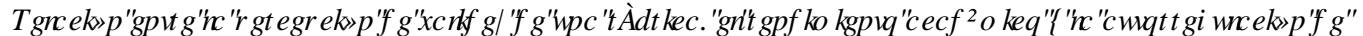 HMXIDQUALGHFHQFDVGHDCHRLUI}

BARNEY, S., KHURUM, M., PETERSEN, K., UNTERKALMSTEINER, M., y JABANGWE, R., (2011). "Improving Students With Rubric-Based Self-Assessment and Oral Feedback" en IEEE Transactions on Education, vol. 55, issue 3, p. 319-325.

DASÍ, A. y IBORRA, M., (2007). "La evaluación de los estudiantes en la Educación Superior.La mejora de la comunicación oral: una perspectiva de proceso". Servei de Formació Permanent. Universitat de València.

DUNBAR, N.E., BROOKS, C.F., y KUBICKA-MILLER, T., (2006). "Oral Communication Skills in Higher Education: Using a Performance-Based Evaluation Rubric to Assess Communication Skills" en Innovative Higher Education, vol. 31, issue 2, p. 115.

ESHUN, E.F. y OSEI-POKU, P., (2013). "Design Students Perspectives on Assessment Rubric in StudioBased Learning" en Journal of University Teaching and Learning Practice, vol. 10, issue 1.

GARCÍA-ROS, R. (2011). "Análisis y validación de una rúbrica para evaluar habilidades de presentación oral en contextos universitarios" en Electronic Journal of Research in Educational Psychology, vol. 9, issue 25 .

JONSSON, A. y SVINGBY, G., (2007). "The use of scoring rubrics: Reliability, validity and educational consequences" en Educational research review, vol. 2, issue 2, p. 130-144.

NICOL, D.J. y MACFARLANE-DICK, D., (2006). "Formative assessment and self-regulated learning: a model and seven principles of good feedback practice" en Studies in Higher Education, vol. 31, issue 2, p. 199-218.

PANADERO, E. y ROMERO, M., (2014). "To rubric or not to rubric? The effects of self-assessment on self-regulation, performance and self-efficacy" en Assessment in Education: Principles, Policy \& Practice, vol. 21 , issue 2 .

PRIEGO QUESADA, J., REQUENA-BUENO, L., JIMÉNEZ-PÉREZ, I., y GARCÍA-ROS, R., (2019). "¿Implicar a los estudiantes en la modificación de rúbricas influye sobre su percepción de validez? " en INRED 2019. V Congreso de Innovación Educativa y Docencia en Red. Editorial Universitat Politècnica de València, 329-342.

REDDY, Y.M. y ANDRADE, H., (2010). "A review of rubric use in higher education" en Assessment \& evaluation in higher education, vol. 35 , isse 4 , p. 435-448.

VERANO-TACORONTE, D., GONZÁLEZ-BETANCOR, S.M., BOLÍVAR-CRUZ, A., FERNÁNDEZMONROY, M., GALVÁN-SÁNCHEZ, I., VERANO-TACORONTE, D., GONZÁLEZ-BETANCOR, S.M., BOLÍVAR-CRUZ, A., FERNÁNDEZ-MONROY, M., y GALVÁN-SÁNCHEZ, I., (2016). "Valoración de la competencia de comunicación oral de estudiantes universitarios a través de una rúbrica fiable y válida" en Revista Brasileira de Educação, vol. 1, issue 64, p. 39-60.

ŽIVKOVIĆ, S., (2014). "The Importance Of Oral Presentations For University Students" en Mediterranean Journal of Social Sciences, vol. 5, issue 19, p. 468. 